\section{Mejoras en la protección de distancia aplicado a fallas con contenido sub-armónicas en líneas de transmisión}

\author{
Javier Antonio Comellys Checa ${ }^{1}$ \\ 1 Universidad Tecnológica de Panamá, \\ jcomellys@gmail.com \\ DOI 10.33412/pri.v11.1.2533
}

\section{(2)(1) $\$(0)$}

Resumen: Las líneas de transmisión con compensación serie capacitiva tienen ventajas técnicas y económicas en los sistemas de Potencia, pero en el momento en que presenta un fenómeno transitorio, las protecciones de distancia se ven afectada en su desempeño, lo que puede originar disparos indeseados en la protección. En esta investigación, se compara el algoritmo de la transformada de Fourier con una variante del método de Prony, para así analizar su desempeño al momento de amortiguar frecuencias sub-armónica, durante una falla eléctrica en una línea de transmisión con compensación serie capacitiva. En esta investigación, se puede observar cómo se mejora la estimación de la impedancia de falla cuando se aplican fallas monofásicas, permitiendo que la protección de distancia mejore su selectividad, al momento de tomar la decisión del disparo. En este documento se analiza un sistema de $400 \mathrm{kV}$ que se desarrolla con el Programa de Transitorio ATP y el análisis de procesamiento de señales se realiza con el lenguaje de programación de Python y Matlab.

Palabras claves: Compensación serie, procesamiento digital de señales, protección de distancia, método de Prony, algoritmos paramétricos, líneas de transmisión con compensación serie capacitiva (LTCSC).

Title: Improved distance protection applied to faults with subharmonic content in transmission lines.

\begin{abstract}
Series compensated transmission lines bring technical and economic advantages for power systems. However, when transient events occur, the performance of distance protection is affected, which can cause undesired tripping in the protection. This study compared the Fourier transform algorithm with a variant of the Prony method, which allows attenuating subharmonic frequencies that are generated at the time of an electrical failure in series compensated transmission lines. It was observed how the estimation of the fault impedance was improved when single-phase faults were applied, allowing the distance protection to improve its selectivity at the moment of making the tripping decision. This study assessed a $400 \mathrm{kV}$ system developed with the alternative transient program was, and the signal
\end{abstract}

processing was assessed using the Python and Matlab programming language.

Key words: Series compensated, digital signal processing, distance protection, Prony method, parametric algorithm, transmission line with capacitive series compensation.

Tipo de artículo: Original

Fecha de recepción: 20 de septiembre de 2019

Fecha de aceptación: 23 de enero de 2020

\section{Introducción}

Los sistemas de potencia se encuentran en una constante evolución como consecuencia de la penetración de la energía renovable a gran escala, el incremento de la generación distribuida y la introducción de nuevas tecnologías que permiten optimizar la capacidad de las líneas de Transmisión. Todo esto conlleva a que las protecciones digitales se enfrenten a nuevos desafíos para mantener los criterios básicos en los sistemas de protecciones eléctricas, como lo es la confiabilidad, sensibilidad, selectividad y velocidad de la protección.

En este contexto, las Líneas de Transmisión con compensación serie capacitiva (LTCSC) han sido una alternativa técnica por mucho tiempo, para aumentar la capacidad de transmisión las Línea de una manera más económica [1,2]. Sin embargo, la presencia de capacitores series en la línea, tiene efectos adversos en los relevadores de protección ya que pueden causar una incorrecta operación en los elementos de distancia y direccional del relevador.

Los relevadores de distancia utilizados para proteger las líneas de transmisión supervisan los parámetros eléctricos de tensión y corriente para así estimar la impedancia de falla al momento en que se origina un transitorio en la línea protegida. Si la protección identifica que las fallas se encuentran dentro de su zona de alcance, el equipo genera el disparo de los interruptores de potencia para asi despejar la falla. Si la falla se encuentra fuera del alcance del relevador, el equipo espera un tiempo para activarse y despejar la falla, actuando como una protección de respaldo.

Con el desarrollo de la tecnología, los relevadores digitales pueden analizar fenómenos transitorios originados en las redes eléctricas de una manera rápida y flexible, ya que se utilizan herramientas matemáticas, de procesamiento digital de señales para analizar las corrientes y tensiones que se generan durante un proceso transitorio. También se tiene la flexibilidad de desarrollar nuevos algoritmos de protección, que pueden trabajar para diferentes condiciones, dependiendo de las características y requerimiento del sistema.

La aplicación de la protección de distancia en líneas compensadas ha generado diferentes líneas de investigación, de forma tal que se han aplicado métodos matemáticos de clasificación de señales para identificar las bajas y altas frecuencias, igualmente se han generado herramientas de filtrado de señales, lo que ha permitido mejorar la capacidad del relevador 
para distinguir entre una falla interna y otras condiciones que pueden ocurrir en el sistema.

Nuevas técnicas de procesamiento digital de señales son de gran interés para los investigadores ya que son la primera etapa que permite emular la señal analógica en forma digital, por tal motivo esta investigación propone una nueva herramienta de medición fasorial que puede ser utilizada en las protecciones de distancia, cuando se aplican a las LTCSC, esto como una nueva alternativa para amortiguar frecuencias que se encuentra debajo de la frecuencia fundamental

En la primera parte de este trabajo se presenta la protección de distancia aplicado a las líneas compensadas, luego se describe los métodos desarrollados para el procesamiento digital de la señal utilizando herramientas de medición fasorial y, por último, se analiza una nueva alternativa para superar los problemas de la protección de distancia cuando se originan frecuencias sub-armónica, producto de una falla transitoria en las LTCSC.

\section{Protección de Distancia en LTCSC}

El relevador de distancia ha sido utilizado desde hace mucho tiempo, para proteger líneas de transmisión de alta tensión ya que tiene la característica de ser selectiva para cubrir una zona determinada y además puede ser utilizada como una protección de respaldo. El relevador estima la impedancia de falla $\mathrm{Z}=\mathrm{U} / \mathrm{I}$ por medio de los parámetros medidos de tensión y corriente desde la ubicación del relevador para así compararlo con una impedancia de falla conocida o de ajuste [3]. Cuando la línea no se encuentra compensada, el alcance en la Zona 1 es normalmente el $80 \%$ de la línea de transmisión y el tiempo de actuación es instantáneo.

En el caso de una falla en el resto de la línea y las líneas adyacentes, se utiliza la Zona 2 y 3 de la protección, cuyo tiempo de activación es temporizado ya que se puede aplicar como una protección de respaldo para las líneas adyacentes de la línea principal a proteger.

Cuando se utiliza la protección de distancia en una LTCSC, se pueden producir problemas en la operación del relevador al momento en que se genera un transitorio en la línea. Algunos de estos problemas están relacionados con la inversión de tensión, la inversión de corriente y la generación de frecuencia de oscilación sub-armónicas [4]. Este último introduce errores en la estimación de la impedancia de falla debido a las oscilaciones que se generan al momento de calcularse su trayectoria, lo que provoca que se demore el disparo de la protección, tal como se observa en la figura 1.

\subsection{Medición Fasorial en LTCSC}

Actualmente, algunos investigadores trabajan en el desarrollo de nuevos algoritmos para el área de protecciones eléctricas, con el fin de afrontar los retos que se presentan cuando se protegen líneas compensadas. En [5,6] se presenta los avances que se han logrado en la protección de líneas con compensación serie capacitiva y las técnicas utilizadas para el procesamiento digital de la señal, como alternativa a la transformada de Fourier.

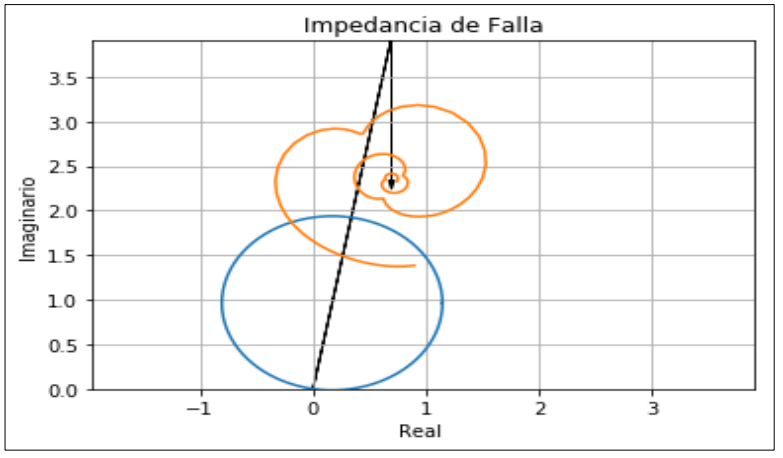

Figura 1. Impedancia vista por el relevador de distancia.

Igualmente, en estos trabajos se presenta otros métodos que permiten la identificación de las fallas utilizando técnicas de inteligencia artificial.

El desempeño de las protecciones en las líneas de transmisión compensadas cuando utilizan el algoritmo de la transformada discreta de fourier (DFT) fue modelado en [7] observándose como las componentes de corrientes subsincrónicas provocan un retardo en la operación del relevador y adicionalmente con la probabilidad de que la trayectoria de la impedancia atraviese zonas de disparo incorrectas. En [8] se utiliza un analizador de redes transitorias (TNA) para el ajuste de relevadores en líneas con compensación serie capacitiva donde se puede comprobar la importancia de los filtros digitales para el buen desempeño de la protección.

También se han investigado otros métodos que permiten aumentar la resolución por medio del modelamiento de la señal, esto son los llamados métodos paramétricos, donde se aplica a señales cuyas frecuencias se encuentran espaciadamente muy cercanas. Entre ellos se encuentra el método de Prony, donde se puede obtener directamente el fasor de la señal de interés sin utilizar ninguna transformada. En [9] se reporta la utilización del Método de Prony, como técnica de filtrado para relevadores de distancias aplicado a líneas compensadas, observándose un tiempo de convergencia de hasta 1.75 ciclos en comparación con un filtro coseno que converge en 3.1 ciclo. En [10] se reporta el uso del Método de Prony como un nuevo método para detectar fallas de altas impedancias, basadas en la extracción de las componentes de la forma de onda de la corriente. El autor llega a la conclusión que este método, introduce una nueva forma para detectar fallas de alta impedancia, el cual puede ser utilizado para desbloquear el relevador de distancia. El algoritmo de Prony ha sido utilizado en [11] y [12] para extraer las características de la señal, en combinación con otros algoritmos de clasificación como las Wavelet y herramientas de aprendizaje [13], donde los resultados obtenidos para ubicar una falla en la línea son más exactos en comparación con otros algoritmos como el método de la onda viajera.

En [14] se mejora la técnica Prony-DFT para estimar y remover transitorios no deseados incluyendo las frecuencias subsincrónicas y las componentes DC en las mediciones de tensión 
y corriente. Con este algoritmo se obtiene una alta exactitud, pero requiere de un tiempo de 3 a 4 ciclos.

\subsection{Estimación fasorial utilizando el Método de Prony.}

La protección de distancia utiliza el fasor de la señal de tensión y corriente para estimar la impedancia de falla. El algoritmo de Fourier es utilizado por las protecciones para estimar los fasores de la señal, pero una de las alternativas que se puede utilizar para estimar los fasores es el método de Prony.

El método de Prony es un método determinístico que consiste en describir la señal como una suma de " $p$ " exponenciales compleja. Una de las ventajas que tiene el método de Prony ante Ios modelos ARMA y AR, es que trata de ajustar la información del modelo en forma determinística y no estocásticamente como estos últimos. Otra de las ventajas de este método, es que nos permite tener una descripción física de la señal en términos de magnitud, fase, frecuencia y amortiguamiento. Este modelo se representa con la siguiente ecuación:

$$
\begin{gathered}
x(n)=\sum_{k=1}^{p} B_{k} \cdot z_{k}^{n}=-\sum_{k=1}^{p} a_{k} \cdot x(n-1) \\
\text { para } n \geq p
\end{gathered}
$$

Las constantes complejas se definen como:

$$
B_{k}=A_{k} \cdot e^{j \theta_{k}} \quad \text { y } \quad z_{k}=e^{\left(\alpha_{k}+j \Omega_{k}\right) \cdot T_{s}}
$$

Donde $T_{s}$ es el periodo de muestreo, $A_{k}$ la amplitud de la $k_{t h}$ exponencial compleja, $\alpha_{k}$ la constante de tiempo en segundos, $\Omega_{k}$ la frecuencia angular en $\mathrm{rad} / \mathrm{s}, \theta_{k}$ el ángulo de fase, $p$ el orden del modelo, $x(n)$ son las muestras de la señal y $\mathrm{a}_{k}$ son los coeficientes del polinomio resultante de la ecuación. De la ecuación anterior se puede observar que el comportamiento de la señal puede ser descrito por un modelo de predicción lineal.

El método de Prony es también llamado método polinomial y su solución se reduce, de un problema no lineal a dos problemas lineales, con una operación no lineal de encontrar las raíces polinómicas. La implementación del método incluye los siguientes pasos:

$\checkmark \quad$ En el primer paso, se estiman los coeficientes del modelo de predicción lineal (LPM). La predicción lineal se representa en forma de matriz con las muestras ordenadas secuencialmente en el tiempo de la señal. Para obtener los coeficientes se puede aplicar algún método estimación de los parámetros AR (Covarianza, Burgs, Yule Walker etc.) o por medio de del método de descomposición de valores singulares.

$\checkmark$ En el segundo paso se realiza la estimación de las raíces del polinomio asociados con el LPM del primer paso $\left(\mathbf{z}_{\boldsymbol{k}}\right)$. En este paso se estima la frecuencia y el factor de amortiguamiento. $\checkmark$ En el último paso, la magnitud y el ángulo de fase de la señal es resuelto con el método de mínimos cuadrados.

En la siguiente imagen se puede observar el proceso que se utiliza para aplicar el método de Prony:

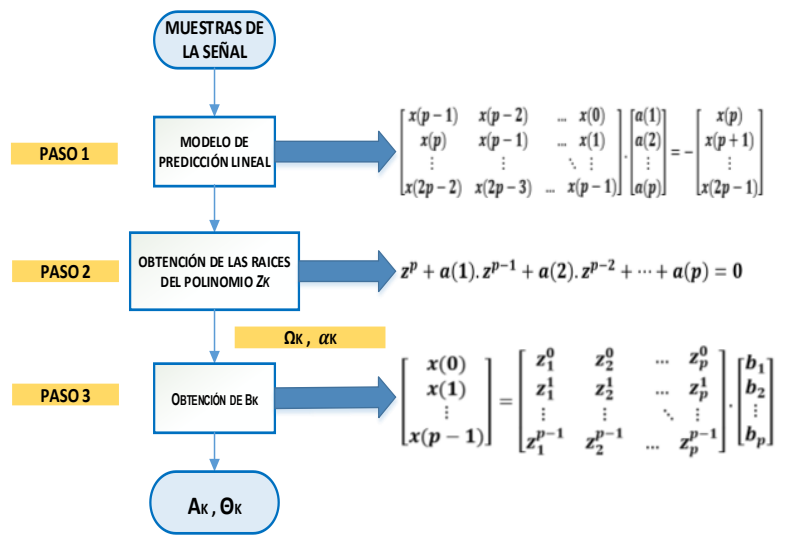

Figura 2. Pasos del método de Prony.

Dentro de los principales inconvenientes que se tienen con el método de Prony, es seleccionar correctamente el orden del modelo, ya que, si el orden del modelo es reducido, se obtiene un espectro muy suavizado, mientras que con un mayor orden se produce picos espurios. Otro de los problemas que se presenta con este método es cuando la señal contiene un ruido considerable ya que puede afectar la estimación del fasor.

\section{Método de Prony Simplificado}

Las señales de tensión y corriente pueden ser analizadas con el método de Prony ya que se puede obtener directamente el fasor de la señal y además puede ser utilizado como un método de filtrado para señales por debajo de la frecuencia fundamental. En [15] se utilizó el método de Prony con mínimos cuadrado, para así estimar los parámetros de la componente sinusoidal. Sin embargo, el método tiene como desventaja que requiere de una carga computacional alta, lo que aumenta el tiempo de procesamiento en un relevador de protección, principalmente cuando se deben obtener las raíces del polinomio característico.

En [16] se propone el método Wavelet-Prony, como una alternativa para mejorar la ubicación de falla.

Como el método de Prony se puede utilizar como una herramienta de filtrado para una determinada frecuencia, se procederá a estimar la impedancia de falla aplicando el método de una manera reducida. Para lograr esto, se supondrá a priori un rango de frecuencia cercana a la señal fundamental de corriente de forma tal que se pueda mejorar la estimación de la impedancia de falla al momento de calcular la trayectoria dentro de la característica Mho tal como se indica en "(3)".

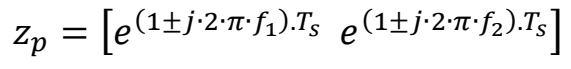


Luego se procede a evaluar el fasor de la señal de corriente por medio de la siguiente formulación matricial:

$$
\left[\begin{array}{c}
x(0) \\
x(1) \\
\vdots \\
x(p-1)
\end{array}\right]=\left[\begin{array}{cccc}
z_{1}^{0} & z_{2}^{0} & \ldots & z_{p}^{0} \\
z_{1}^{1} & z_{2}^{1} & \ldots & z_{p}^{1} \\
\vdots & \vdots & \ddots & \vdots \\
z_{1}^{p-1} & z_{2}^{p-1} & \ldots & z_{p}^{p-1}
\end{array}\right] \cdot\left[\begin{array}{c}
b_{1} \\
b_{2} \\
\vdots \\
b_{p}
\end{array}\right]
$$

Una vez que se obtiene la pseudo-inversa de $\mathbf{z}_{p}$ y los valores de $\boldsymbol{b}_{\boldsymbol{p}}$ se puede estimar de manera directa la amplitud y ángulo del fasor de la impedancia de falla (3).

Para el caso de fallas que se encuentren cercanas al banco de capacitores se supondrá que las protecciones del banco de capacitores actuarán en forma instantánea para proteger al banco de capacitores de forma tal que desaparece el efecto capacitivo en serie con la línea.

\section{Modelo de prueba del sistema a analizar}

Para evaluar el desempeño del método, se procede a analizar un sistema de $400 \mathrm{kV}$ a una frecuencia nominal de $50 \mathrm{~Hz}$. El sistema comprende de dos líneas de transmisión, una de $300 \mathrm{~km}$ y la segunda de $150 \mathrm{~km}$. Los parámetros de ambas líneas son las siguientes:

Tabla 1. Características de la línea de transmisión.

\begin{tabular}{|c|c|c|}
\hline $\mathbf{z 1}$ & $0.0267+j 0.3142$ & {$[\Omega / \mathrm{Km}]$} \\
\hline $\mathbf{z 0}$ & $0.275+\mathrm{j} 1.0264$ & {$[\Omega / \mathrm{Km}]$} \\
\hline $\mathbf{c 1}$ & 0.013 & {$[\mu \mathrm{F} / \mathrm{Km}]$} \\
\hline $\mathbf{c 0}$ & 0.0085 & {$[\mu \mathrm{F} / \mathrm{Km}]$} \\
\hline
\end{tabular}

En la figura 3, se presenta el diagrama unifilar del sistema modelado, desarrollado en el programa de transitorios ATP:

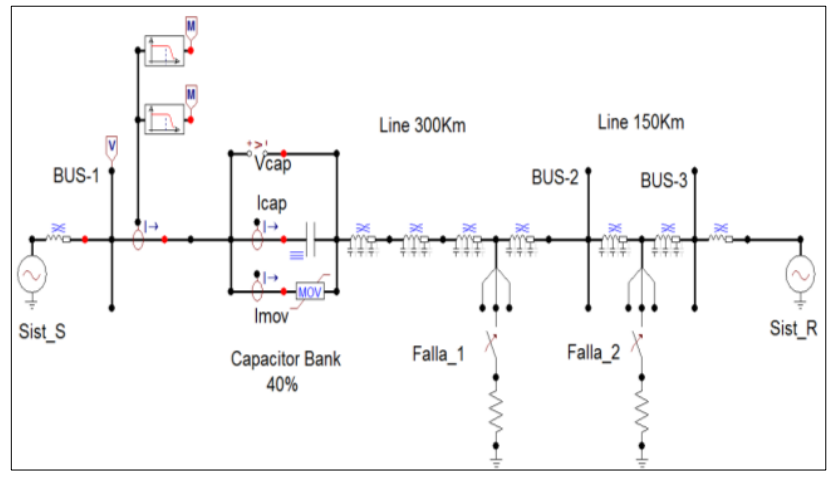

Figura 3. Diagrama Unifilar del sistema de 400kV en ATP.
El modelo comprende un banco de capacitores con una compensación serie del $60 \%$ de la inductancia de línea de transmisión. Durante la simulación, se generan fallas de fase a tierra a una distancia de $80 \%$ de la línea de transmisión entre el bus 1 y bus 2 y fallas al $110 \%$ ubicado en la línea adyacente entre el bus 2 y bus 3 . Los datos generados de cada una de las fallas se procesan utilizando el nuevo método de Prony y se compara con el algoritmo de Fourier.

\section{Pruebas y Resultados}

En esta sección se analizará el desempeño del algoritmo aquí propuesto. La protección de distancia en la figura 3 se ubica en el Bus 1, y se ajusta para que la Zona 1 cubra el $80 \%$ de la línea 1 y la Zona 2 se ajusta al $100 \%$ de la línea 1 más $20 \%$ de la siguiente línea. Para este estudio se genera fallas monofásicas en medio de la línea de transmisión y al final de la línea y se considera que la línea se encuentra compensada a un $60 \%$ de la inductancia de la línea por medio de un banco de capacitores.

Cuando se realizan fallas a la mitad de la línea de transmisión y al final de la línea, se puede observar que en este último caso la frecuencia sub-armónica tiene un mayor impacto en la forma de onda figura 4 (a), por tal motivo se considera este caso para ser analizado con el nuevo método.

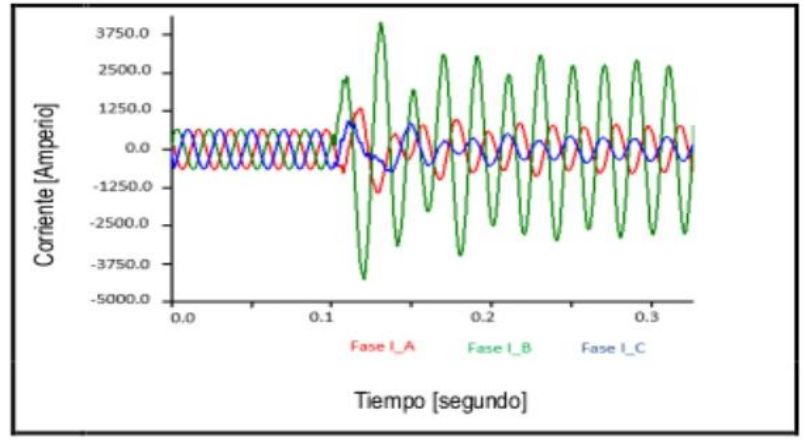

(a)

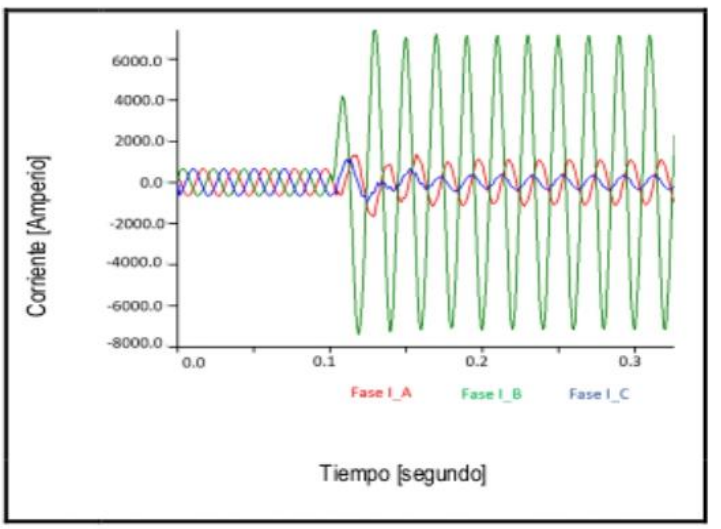

(b)

Figura 4. Falla monofásica a) A inicio de la línea de transmisión de (150 km), b) En medio de la línea de transmisión $300 \mathrm{~km}$. 
El proceso de adquisición de los datos de corriente y tensión de falla se obtiene con el programa ATP, luego se procede a realizar la conversión análogo-digital a una tasa de muestreo de $1600 \mathrm{~Hz}$ para así analizar el fasor de la señal aplicando las herramientas de Fourier y el método de Prony tal como se indica en la figura 5.

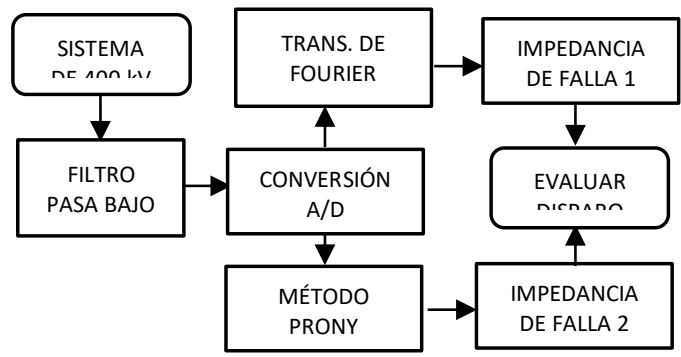

Figura 5. Etapas de procesamiento de la señal de tensión y corriente.

Durante las simulaciones se puede observar que las fallas que se generan en el extremo de la línea de trasmisión y están lejanas al banco de capacitores, pueden originar señales por debajo de la frecuencia fundamental lo que provoca que la protección de distancia inicie un disparo en la Zona 1, a pesar de que el origen de la falla está en la Zona 2. En la figura 6 (a) se puede observar que la señal de corriente de falla, aplicando la DFT, muestra valores por encima de 4kA y la señal de corriente está distorsionada. Al aplicar el nuevo método de Prony figura 6 (b) la oscilación en amplitud de corriente se reduce a 3.5kA y la forma de la señal se observa amortiguada.

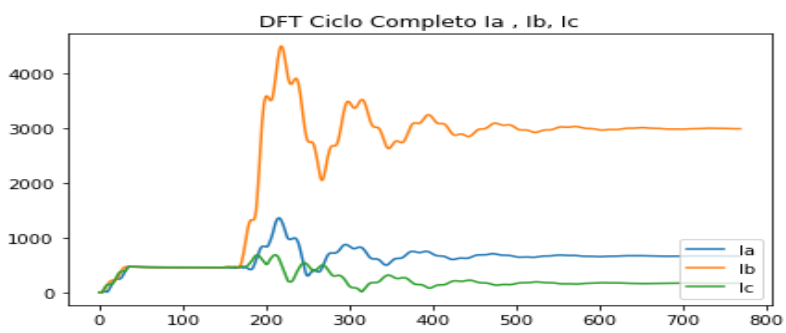

(a)

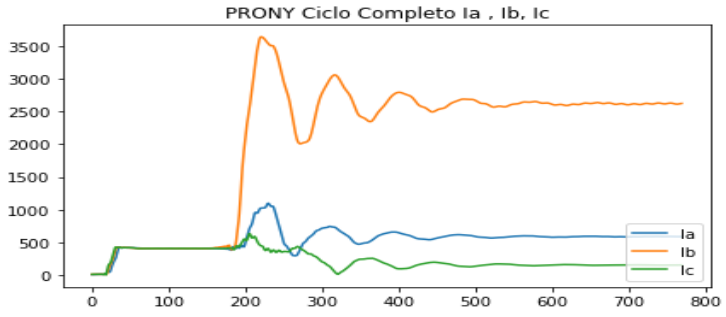

(b)

Figura 6. Señal de corriente utilizando a) DFT, b) Método de Prony.
En la figura 7 se puede observar, como la trayectoria de la impedancia de falla pasa por la Zona 1 cuando se estima la impedancia con la DFT, lo que origina un disparo instantáneo.

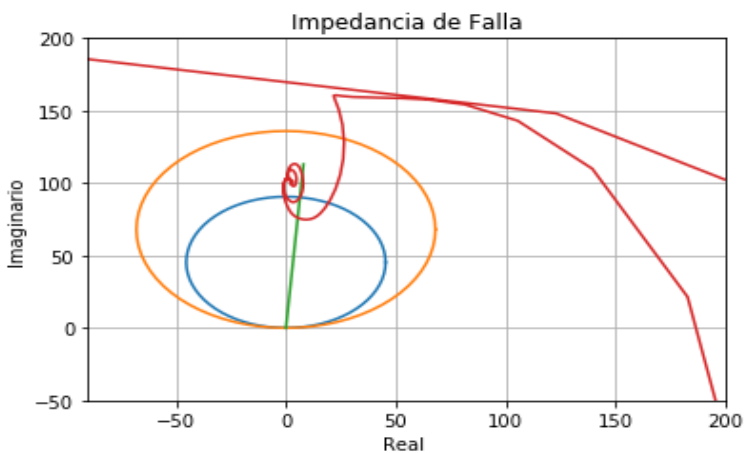

Figura 7. Trayectoria de la Impedancia de Falla utilizando la DFT.

En la figura 8 se puede observar la trayectoria de la impedancia de falla cuando se aplica el método de Prony mejorado.

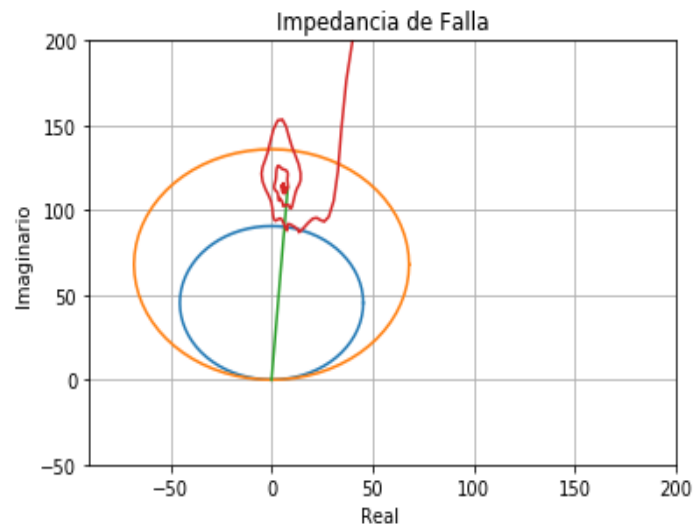

Figura 8. Trayectoria de la Impedancia de Falla utilizando el Método de Prony.

En este último caso, la trayectoria de la impedancia de falla se mantiene en la Zona 2, ubicando la falla en el lugar donde se origina la falla sin pasar por la Zona 1 de disparo instantáneo.

\section{Conclusión}

En esta investigación se desarrolla un modelo que incluye una línea de transmisión, un banco de capacitores con sus elementos de protección. El modelo desarrollado en ATP, se utiliza para simular fallas de fase a tierra y así analizar y procesar las señales de tensión y corriente con el algoritmo de Prony en su forma reducida.

Durante la simulación se pudo observar que las señales de corrientes pueden presentar oscilaciones por debajo de la frecuencia fundamental, especialmente cuando las fallas se 
encuentran al final de la línea de transmisión, lejos del capacitor serie. En el caso de que la falla se encuentre cercano al banco de capacitores, la protección MOV actúa, lo que provoca una distorsión en los puntos máximos de la forma de onda de tensión y corriente, pero no se observa oscilaciones sub-armónicas, por lo tanto, este estudio solo considera fallas lejanas al banco de capacitores que es cuando hay una mayor probabilidad de que se generen oscilaciones por debajo de la frecuencia fundamental.

En esta investigación se desarrolla un nuevo método que permite obtener la magnitud y ángulo del fasor de corriente y tensión por medio de la herramienta de Prony en su forma reducida. EL método de Prony, en su forma reducida, puede ser aplicado en la protección de distancia ya que se disminuye la cantidad de operaciones matemáticas que se deben realizar en comparación con el método de Prony original.

Se realiza una comparación entre el algoritmo de Fourier y el nuevo método de Prony observándose que este último logra amortiguar la señal sub-armónica, estabilizando la señal de corriente de una manera más rápida, de igual forma las señales que se obtiene con el nuevo método tiene una forma más suavizada en comparación con el algoritmo de Fourier.

Por último, se pudo comprobar que con el nuevo método la trayectoria de la impedancia de falla en la característica Mho no cruza la zona 1 de disparo instantáneo a pesar de que el origen de la falla se encuentra en la zona 2 de disparo, actuando correctamente la protección, por lo tanto, el nuevo método mejora la confiabilidad de la protección.

\section{Referencias}

[1] L. Kirschner, M. Correia, J. Eduardo, C. Fernandes, R. Miinchmeier, and I. Introduction, "Benefits and Design Aspects of Sao Sao Joao do Piaui 500 kV Series Capacitors," pp. 1-5, 2008.

[2] G. Corpuz, K. Koellner, J. Bell, S. Rajan, A. Somani, and M. Thompson, "Series-compensated line protection challenges in the CREZ region," in 2014 67th Annual Conference for Protective Relay Engineers, 2014, pp. 664-675.
[3] L. Kirschner, M. Correia, J. Eduardo, C. Fernandes, R. Miinchmeier, and I. Introduction, "Benefits and Design Aspects of Sao Sao Joao do Piaui 500 kV Series Capacitors," pp. 1-5, 2008.

[4] G. Ziegler, Numerical Distance Protection: Principles and Applications Wiley, 2006

[5] H. J. Altuve, J. B. Mooney, and G. E. Alexander, "Advances in seriescompensated line protection," in 2009 62nd Annual Conference for Protective Relay Engineers, 2009, pp. 263-275.

[6] B. Vyas, R. P. Maheshwari, and B. Das, "Protection of series compensated transmission line: Issues and state of art," Electr. Power Syst. Res., vol. 107, pp. 93-108, Feb. 2014.

[7] C. S. Yu, J. Z. Yang, and C. W. Liu, "New Fourier filter design for fault current filtering of series compensated lines," Int. J. Power Energy Syst., vol. 28, no. 2, pp. 203-210, 2008.

[8] E. Orduña, D. G. Colome, G. D. Guidi, G. Ratta, O. M. Torres, and J. S. Ulloa, "Behavior of the Digital Fourier Transform as Numerical filter in Distance Protection of Series Compensated Transmission Lines. Simulations With a Real Transmission System," IEEE Lat. Am. Trans., vol. 10, no. 5, pp. 2080-2087, Sep. 2012.

[9] O. Trad, G. Ratta, and M. Torres, "Experiences in Setting Protection of Series Capacitor Compensated Lines," Proceedings of the International Conference on Power System Transients (IPST01), pp. 1-6, June 2001.

[10] L. A. Trujillo G, A. Conde E, and Z. Leonowicz, "Application of the Prony method for compensation of errors in distance relays," in 2013 12th International Conference on Environment and Electrical Engineering, 2013, pp. 568-572.

[11] Abilash Thakallapelli, Veermata Jijabai Technological Institute, "Detection of High Impedance Faults by Distance Relays using Prony's Method", International Journal of Advanced Technology \& Engineering Research (IJATER), 2012.

[12] M. Farshad and J. Sadeh, "Transmission line fault location using hybrid wavelet-Prony method and relief algorithm," Int. J. Electr. Power Energy Syst., vol. 61, pp. 127-136, Oct. 2014.

[13] M. M. Tawfik and M. M. Morcos, "ANN-based techniques for estimating fault location on transmission lines using Prony method," IEEE Trans. Power Deliv., vol. 16, no. 2, pp. 219-224, Apr. 2001.

[14] T. P. S. Bains and M. R. Dadash Zadeh, "Enhanced Phasor Estimation Technique for Fault Location in Series-Compensated Lines," IEEE Trans. Power Deliv., vol. 30, no. 4, pp. 2058-2060, Aug. 2015.

[15] Zygarlicki J, Mroczka J, "Variable-frequency Prony method in the analysis of electrical power quality," Metrology and Measurement Systems.,2012 vol: 19 (1) pp: 39-48.

[16] Farshad M, Sadeh J, "Transmission line fault location using hybrid waveletProny method and relief algorithm," International Journal of Electrical Power \& Energy Systems., 2014 vol: 61 pp: 127-136 